\title{
$\mathrm{Co}_{n}(\boldsymbol{n}=\mathbf{2} \sim \mathbf{1 0})$ 团簇的结构和磁性 *
}

\author{
吕瑾许小红武海顺 \\ (山西师范大学化学与材料科学学院, 临汾 041004)
}

\begin{abstract}
摘要 采用密度泛函理论中的局域自旋密度近似 (LSDA) 和广义梯度近似 (GGA) 对 $\mathrm{Co}_{n}(n=2 \sim 10)$ 团簇的几何 构型进行优化, 并对能量、频率和磁性进行了计算, 两种方法确定的基态构型完全一致, 并从平均键长、平均配位 数和对称性对磁性的影响进行了理论探讨. 研究表明, $\mathrm{Co}_{n}(n=2 \sim 10)$ 基态团簇的磁性在 $n=2 \sim 4$ 时主要受平 均键长的影响, 在 $n=5 \sim 9$ 时主要受平均配位数的影响, 在 $n=10$ 时受原子间距和平均配位数的相互影响, 最 终导致与 $\mathrm{Co}_{8}$ 基态团簇具有相同的磁性 . 基态团簇在 $\mathrm{Co}_{5}$ 和 $\mathrm{Co}_{9}$ 出现了磁性局域最小点.
\end{abstract}

关键词: 团簇，基态构型，磁矩，平均键长，平均配位数，对称性 中图分类号: 0664

过渡金属团簇在开发新磁性材料和新催化剂 领域具有广阔的应用前景。对于铁族团簇体系, 理 论 ${ }^{[1-6]}$ 和实验 ${ }^{[7-9]}$ 研究表明, 它们具有比相应的体材 料更强的磁性。前人已经做了大量理论研究。如 Mlynarski 等 ${ }^{[10]}$ 用密度泛函理论(DFT) 中非局域广 义梯度近似 $(\mathrm{GGA})$ 对 $\mathrm{Co}_{n}(n \leqslant 6,13)$ 的团簇进行 了计算, 就团簇的对称性对磁矩的增强作了分析. Fan 等 ${ }^{[11]}$ 利用 $\mathrm{ADF}$ 对 $\mathrm{Co}_{n}(n=2 \sim 8)$ 进行了几何、 电子结构和磁性质的研究, 确定了它们的基态构 型, 并指出 $\mathrm{CO}_{6}$ 团簇具有超常的稳定性, 而且在 $\mathrm{Co}_{n}$ $(n=2 \sim 8)$ 团簇中没有磁幻数出现. $\mathrm{Li}$ 等 ${ }^{[12]}$ 对 $\mathrm{Co}_{13}$ 的研究指出, 团簇的对称性越高, 磁性就越大. Duan 等 ${ }^{[13]}$ 在对 $\mathrm{Fe}_{55} 、 \mathrm{Co}_{55}$ 和 $\mathrm{Ni}_{55}$ 团簇的对称性和磁性研 究中指出对称性对磁性的影响与团簇的大小有关. 大量研究表明, 团簇的磁性与其对称性、平均配位 数和平均键长有关 ${ }^{[6,10,12-14]}$. 用 DFT 中 GGA 和 LS$\mathrm{DA}$ 方法对 $\mathrm{Co}_{n}(n=7 \sim 10)$ 团簇的研究尚未见报 道. 本文将在优化 $\mathrm{Co}_{n}(n=2 \sim 10)$ 团簇构型的基础 上, 从对称性、平均配位数和平均键长等角度对钴 团簇的结构和磁性进行系统的研究. 所得结论不 仅对探索钴团簇向体材料的演变过程具有重要的 意义, 而且对新磁性材料的研制也有很重要的理论 意义。

\section{1 计算方法}

采用 DFT 下的局域自旋密度近似(LSDA) 和广 义梯度近似 (GGA), 用 $\mathrm{DmoL}^{3}$ 软件包对全部构型 进行优化和电子结构计算。在 LSDA 中, 对交换相 关势的修正选择 $\mathrm{VWN}^{[15]}$ 函数; 在 $\mathrm{GGA}$ 中, 选择 Becke ${ }^{[16]}$ 的交换梯度修正和 Perdew-Wang ${ }^{[17]}$ 的关联 梯度修正. 所有的计算均在 FINE 网格下完成. 采 用带极化的双数值原子基组 (DNP) 进行全电子计 算, 自洽过程以体系的能量和电荷密度分布是否收 玫为依据, 精度均优于 $10^{-6}$ a. u., 梯度和位移的收 敛精度优于 $10^{-2}$ a. u. $\cdot \mathrm{nm}^{-1}$ 和 $5 \times 10^{-4} \mathrm{~nm}$, 能量 的收玫精度优于 $10^{-5}$ a. u. ; 另外, 在计算中, 我们在 缺省轨道占据 Smearing 参数下, 对 $\mathrm{Co}_{n}(n=2 \sim 10)$ 团簇的所有几何构型进行优化, 并对能量和磁性进 行计算, 在 Smearing 参数为 0.03 a. u. 时计算其频 率, 其中 $\mathrm{GGA}$ 方法下 $D_{3 h}$ 对称性的 $\mathrm{Co}_{9}$ 团簇和 $D_{2 h}$ 对称性的 $\mathrm{CO}_{10}$ 团簇在 Smearing 参数为 0.04 a. u. 时计算其频率. 全部计算工作在山西师范大 学材料化学研究所完成.

\section{2 结果与讨论}

\section{1 团簇的基态几何构型}

图 1 给出了 GGA 和 LSDA 下优化得到的 $\mathrm{Co}_{n}$ $(n=2 \sim 10)$ 团簇的基态结构. $n \leqslant 3$ 为线性和平面 构型, $n \geqslant 4$ 为紧密堆积的三维结构. 表 1 列出了 $\mathrm{Co}_{n}(n=2 \sim 7)$ 基态几何结构的对称性和几何参数, 


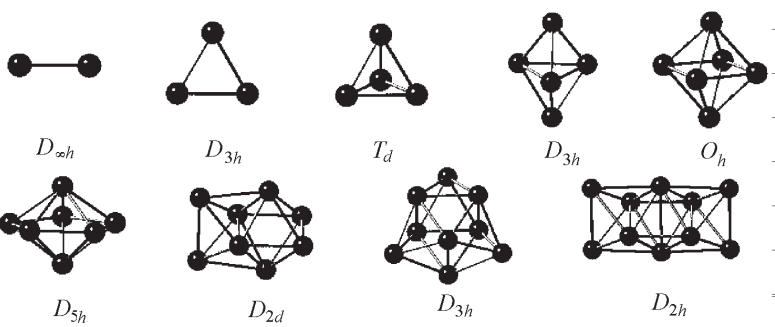

图 $1 \operatorname{Co}_{n}(n=2 \sim 10)$ 团簇的基态几何结构

Fig. 1 Geometric configuration of the ground state structures of $\operatorname{Co}_{n}(n=2 \sim 10)$ clusters

表 $1 \operatorname{Co}_{n}(n=2 \sim 7)$ 基态几何构型的对称性和几何参数 Table 1 Symmetry and geometric parameters of the ground state structures of $\mathrm{Co}_{n}(n=2 \sim 7)$ clusters

\begin{tabular}{ccclcccc}
\hline \multicolumn{3}{c}{ GGA } & & \multicolumn{3}{c}{ LSDA } \\
\cline { 1 - 4 } \cline { 5 - 7 } Cluster & Sym. & $l / \mathrm{nm}$ & & Cluster & Sym. & $l / \mathrm{nm}$ \\
\hline $\mathrm{Co}_{2}$ & $D_{\infty h}$ & 0.2125 & & $\mathrm{Co}_{2}$ & $D_{\infty h}$ & 0.1949 \\
$\mathrm{Co}_{3}$ & $D_{3 h}$ & 0.2386 & & $\mathrm{Co}_{3}$ & $D_{3 h}$ & 0.2172 \\
$\mathrm{Co}_{4}$ & $T_{d}$ & 0.2295 & & $\mathrm{Co}_{4}$ & $T_{d}$ & 0.2234 \\
$\mathrm{Co}_{5}$ & $D_{3 h}$ & $a=0.2383$ & & $\mathrm{Co}_{5}$ & $D_{3 h}$ & $a=0.2271$ \\
& & $b=0.2312$ & & & & $b=0.2239$ \\
$\mathrm{Co}_{6}$ & $O_{h}$ & 0.2303 & & $\mathrm{Co}_{6}$ & $O_{h}$ & 0.2247 \\
$\mathrm{Co}_{7}$ & $D_{5 h}$ & $a=0.2232$ & & $\mathrm{Co}_{7}$ & $D_{5 h}$ & $a=0.2172$ \\
& & $b=0.2393$ & & & & $b=0.2330$ \\
\hline
\end{tabular}

图 2 给出了 $\mathrm{Co}_{n}(n=8 \sim 10)$ 基态团簇的几何参数.

$\mathrm{Co}_{2}$ 团簇基态构型为 $D_{\infty h}$ 对称性, 钴原子间为 典型的 $\delta$ 键. 就 $\mathrm{Co}_{3}$ 团簇, 对同一对称性不同键长 的构型进行优化和能量计算, GGA 和 LSDA 给出的 基态均为 $D_{3 h}$ 对称性, 键长分别为 $0.2386 \mathrm{~nm}$ (GGA) 和 $0.2172 \mathrm{~nm}$ (LSDA). $\mathrm{Co}_{4}$ 和 $\mathrm{Co}_{6}$ 团簇的基 态几何构型分别为典型正四面体和正八面体, $\mathrm{CO}_{5}$ 和 $\mathrm{Co}_{7}$ 团簇分别为紧密堆积的正三角双雉和正五三 角双雉, 它们的几何参数见表 $1 . \mathrm{Co}_{8} 、 \mathrm{Co}_{10}$ 和 $\mathrm{Co}_{9}$ 团 簇的基态构型分别是在 $\mathrm{CO}_{6}$ 团簇八面体和三棱柱的 基础上加了几个帽原子, 几何参数如图 2 所示 . 总 之, GGA 和 LSDA 两种方法所确定的基态构型完全 相同. 而且我们所确定的 $\mathrm{Co}_{n}(n=2 \sim 6)$ 团簇基态 的对称性与 Mlynarski 等 ${ }^{[10]}$ 结果完全一致.与 Fan

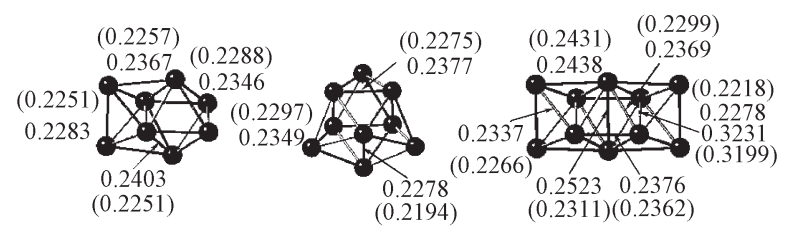

图 $2 \mathrm{Co}_{8} 、 \mathrm{Co}_{9}$ 和 $\mathrm{Co}_{10}$ 基态团簇的键长

Fig. 2 Bond length of the ground state structures of $\mathrm{Co}_{8}, \mathrm{Co}_{9}$ and $\mathrm{Co}_{10}$ clusters the LSDA value in bracket
等 ${ }^{[11]}$ 的结果在 $n=4 、 5 、 8$ 时出现了差别. 我们所 确定的 $\mathrm{Co}_{n}(n=5 \sim 8)$ 的对称性与 Bouarab 等 ${ }^{[18]}$ 所 研究的相应 $\mathrm{Ni}_{n}$ 结果一致。

\section{2 团簇的磁性}

在 $\mathrm{Co}_{n}(n=2 \sim 10)$ 团簇的磁性计算中, 我们所 得磁矩与先前的理论结果一样, 除 LSDA 给出的 $\mathrm{Co}_{9}$ 团簇的平均磁矩为 $1.67 \mu_{\mathrm{B}}\left(1 \mu_{\mathrm{B}}=1.165 \times 10^{-29}\right.$ $\mathrm{Wb} \cdot \mathrm{m})$ 外, 均大于钴体材料磁矩 $1.76 \mu_{\mathrm{B}}$. 团簇的 局域磁矩列于表 2 . 为了更好地研究影响团簇磁性 的因素, 根据公式 $\bar{m}=1 / N \sum_{i} m_{i}$ 计算了每个团簇的平 均磁矩见表 3 . 当 $n=2 、 3 、 4 、 6$ 时, 平均磁矩与文献 [10-11, 19]完全吻合, $n=7 、 8$ 时, 与文献 [11]完全 吻合, 仅在 $n=5$ 时出现了差别. 对于 $\mathrm{Co}_{5}$ 团簇, GGA 的平均磁矩为 $2.20 \mu_{\mathrm{B}}$, 与 Andriotis 等 ${ }^{[19]}$ 的结 果一致, LSDA 为 $1.80 \mu_{\mathrm{B}}$. 相对于其它团簇, GGA 和 LSDA 给出的 $\mathrm{Co}_{5}$ 基态磁性出现了差别,一方面 是由于 $\mathrm{GGA}$ 在计算中低估结合能, 高估键长; LSDA

\section{表 $2 \operatorname{Co}_{n}(n=2 \sim 10)$ 团簇的局域磁矩 $(m)$}

Table 2 Local magnetic moments $(m)$ of the ground state structures of $\mathrm{Co}_{n}(n=2 \sim 10)$ clusters

\begin{tabular}{|c|c|c|c|c|c|c|c|c|}
\hline \multirow{2}{*}{ Cluster } & \multicolumn{2}{|c|}{ GGA LSDA } & \multirow{2}{*}{ Cluster } & \multicolumn{2}{|c|}{ GGA LSDA } & \multirow{2}{*}{ Cluster } & \multicolumn{2}{|c|}{ GGA LSDA } \\
\hline & \multicolumn{2}{|c|}{$m / \mu_{\mathrm{B}}$} & & \multicolumn{2}{|c|}{$m / \mu_{\mathrm{B}}$} & & \multicolumn{2}{|c|}{$m / \mu_{\mathrm{B}}$} \\
\hline \multirow[t]{2}{*}{$\mathrm{Co}_{2}\left(D_{\infty h}\right)$} & 2. 00 & 2.00 & $\mathrm{Co}_{7}\left(D_{5-h}\right)$ & 2.09 & 2.11 & $\mathrm{Co}_{9}\left(D_{3 h}\right)$ & 1. 80 & 1.80 \\
\hline & 2.00 & 2.00 & & 2.27 & 2. 22 & & 1. 41 & 1. 41 \\
\hline \multirow[t]{3}{*}{$\mathrm{Co}_{3}\left(D_{3 h}\right)$} & 2. 33 & 2.33 & & 2.09 & 2. 11 & & 1. 80 & 1. 80 \\
\hline & 2.33 & 2.33 & & 2.09 & 2. 11 & & 1.80 & 1. 80 \\
\hline & 2.33 & 2.33 & & 2.09 & 2. 11 & & 1. 41 & 1. 41 \\
\hline \multirow[t]{4}{*}{$\mathrm{Co}_{4}\left(T_{d}\right)$} & 2.50 & 2.50 & & 2.09 & 2.11 & & 1. 41 & 1. 41 \\
\hline & 2.50 & 2.50 & & 2.27 & 2.22 & & 1. 80 & 1. 80 \\
\hline & 2.50 & 2.50 & $\mathrm{Co}_{8}\left(D_{2 d}\right)$ & 2. 03 & 2.04 & & 1. 80 & 1. 80 \\
\hline & 2.50 & 2.50 & & 1. 98 & 1. 96 & & 1. 80 & 1. 80 \\
\hline \multirow[t]{5}{*}{$\mathrm{Co}_{5}\left(D_{3 h}\right)$} & 2. 18 & 1.76 & & 2.03 & 2.04 & $\mathrm{Co}_{10}\left(D_{2 h}\right)$ & 1.90 & 1.83 \\
\hline & 2.22 & 1.87 & & 1. 98 & 1. 96 & & 1. 92 & 1.95 \\
\hline & 2. 18 & 1.76 & & 2. 03 & 2.04 & & 1. 92 & 1. 95 \\
\hline & 2. 22 & 1.87 & & 1. 98 & 1.96 & & 2.13 & 2.14 \\
\hline & 2. 18 & 1.76 & & 2.03 & 2. 04 & & 2.13 & 2. 14 \\
\hline \multirow[t]{6}{*}{$\mathrm{Co}_{6}\left(\mathrm{O}_{h}\right)$} & 2.33 & 2.33 & & 1. 98 & 1.96 & & 1.92 & 1. 95 \\
\hline & 2.33 & 2.33 & & & & & 1. 92 & 1.95 \\
\hline & 2.33 & 2.33 & & & & & 1. 90 & 1. 82 \\
\hline & 2.33 & 2.33 & & & & & 2.13 & 2.14 \\
\hline & 2.33 & 2.33 & & & & & 2. 13 & 2. 14 \\
\hline & 2. 33 & 2. 33 & & & & & & \\
\hline
\end{tabular}

$1 \mu_{\mathrm{B}}=1.165 \times 10^{-29} \mathrm{~Wb} \cdot \mathrm{m}$ 
表 $3 \operatorname{Co}_{n}(n=2 \sim 10)$ 团簇的平均磁矩 $\left(\bar{m} / \mu_{\mathrm{B}}\right)$

Table 3 Average magnetic moment $\left(\bar{m} / \mu_{\mathrm{B}}\right)$ of the ground state structures of $\mathrm{Co}_{n}(n=2 \sim 10)$ clusters

\begin{tabular}{cccccccccc}
\hline & $\mathrm{Co}_{2}$ & $\mathrm{Co}_{3}$ & $\mathrm{Co}_{4}$ & $\mathrm{Co}_{5}$ & $\mathrm{Co}_{6}$ & $\mathrm{Co}_{7}$ & $\mathrm{Co}_{8}$ & $\mathrm{Co}_{9}$ & $\mathrm{Co}_{10}$ \\
\hline Our work (GGA) & 2.00 & 2.33 & 2.50 & 2.20 & 2.33 & 2.14 & 2.00 & 1.67 & 2.00 \\
(LSDA) & 2.00 & 2.33 & 2.50 & 1.80 & 2.33 & 2.14 & 2.00 & 1.67 & 2.00 \\
Literature [10] & 2.00 & 2.33 & 2.50 & 2.60 & 2.33 & & & & \\
Literature [11] & 2.00 & 2.33 & 2.50 & 2.60 & 2.33 & 2.14 & 2.00 & \\
Literature [19] & 2.00 & 2.33 & 2.50 & 2.20 & 2.33 & & & & \\
\hline
\end{tabular}

在计算中高估结合能, 低估键长, 使 $\mathrm{Co}_{5}$ 团簇的键长 差别 $\Delta a=0.0112 \mathrm{~nm}, \Delta b=0.0073 \mathrm{~nm}$, 明显大于 $\mathrm{Co}_{4}$ 的 $0.0061 \mathrm{~nm}$ 和 $\mathrm{Co}_{6}$ 的 $0.0056 \mathrm{~nm}$. 另一方面 $\mathrm{CO}_{5}$ 中 5 个原子有两种配位环境, 而 $\mathrm{CO}_{4}$ 和 $\mathrm{CO}_{6}$ 的 所有原子配位环境相同, 键长的较大变化对 $\mathrm{Co}_{5}$ 的 局域环境有很大的改变, 二者又是影响磁性的主要 因素, 致使磁性出现差异. 从表 3 可以看出, 随着 $n$ 的增大, 团簇磁性从 $\mathrm{Co}_{2}$ 到 $\mathrm{Co}_{4}$ 在逐渐地增大, 从 $\mathrm{CO}_{4}$ 到 $\mathrm{CO}_{8}$ 在逐渐地减小, $\mathrm{CO}_{8}$ 和 $\mathrm{CO}_{10}$ 具有相同的平 均磁矩, 在 $\mathrm{Co}_{5}$ 和 $\mathrm{CO}_{9}$ 出现了磁性的局域最小点. 对 称性是影响团簇磁性的一个很重要的因素. Dunlap 和 $\mathrm{Li}^{[6,12]}$ 在对 $\mathrm{Fe}_{13}$ 和 $\mathrm{Co}_{13}$ 团簇的研究中指出, 团簇的 对称性越高, 磁性就越大. 通常团簇的对称性越高, 局域 $d$ 电子态就越窄, 越容易导致自旋擘裂, 从而形 成较多自旋平行的 $d$ 电子, 产生较大磁性. 如 $D_{3 h}$ 的 $\mathrm{Co}_{3}, T_{d}$ 的 $\mathrm{Co}_{4}$ 和 $O_{h}$ 的 $\mathrm{Co}_{6}$ 与其对称性较低的异 构体相比, 磁性均为最大.

\section{3 团簇的磁性与平均键长和平均配位数的关系}

过渡金属团簇的磁性主要受原子配位数和平均 键长的影响. 团簇内原子配位数越小, 团簇的局域 $d$ 电子态越窄越容易发生自旋䢃裂, 从而有较多自 旋平行的 $d$ 电子, 形成较大的磁矩. 随着平均配位 数的增大, 局域 $d$ 电子态在加宽, 自旋䢃裂减少, 磁 矩变小. 团簇平均键长越大, 原子间的波函数交叠 越少, 越能保持自由原子的磁矩, 磁矩越大. 表 4 给 出了 $\mathrm{Co}_{n}(n=2 \sim 10)$ 基态团簇的平均配位数和平 均键长, 平均配位数随着团簇的增大, 在逐渐地增 大. 对于平均键长, $\mathrm{GGA}$ 下从 $\mathrm{Co}_{2}$ 到 $\mathrm{Co}_{3}$ 明显增大, 到了 $\mathrm{Co}_{4}$ 又有所降低, 但不影响从 $\mathrm{Co}_{2}$ 到 $\mathrm{Co}_{4}$ 的增
大趋势, $\mathrm{LSDA}$ 下从 $\mathrm{Co}_{2}$ 到 $\mathrm{Co}_{4}$ 在逐渐的增大, 从 $\mathrm{CO}_{5}$ 到 $\mathrm{Co}_{9}$ 两种方法下的平均键长都几乎不变, $\mathrm{CO}_{10}$ 又明显增大. 平均配位数和平均键长的这种变化与 团簇磁性的变化相符. 平均键长和平均配位数对磁 性的影响存在一定的竞争, 如 $\mathrm{Co}_{2}$ 到 $\mathrm{Co}_{4}$ 平均配位 数从 1 到 3 , 磁性应该在逐渐地减小, 而实际上却在 增大, 这说明磁性主要受键长的影响. $\mathrm{CO}_{5}$ 团簇的基 态出现了磁性局域最小点. 从 $\mathrm{CO}_{6}$ 到 $\mathrm{Co}_{9}$ 磁性在逐 渐减小, 平均配位数在逐渐增大, 而平均键长几乎不 变, 说明这些团簇的磁性主要受配位数的影响. $\mathrm{Co}_{8}$ 和 $\mathrm{Co}_{9}$ 比较, $\mathrm{Co}_{8}$ 的磁矩为 $2.00 \mu_{\mathrm{B}}, \mathrm{Co}_{9}$ 为 $1.67 \mu_{\mathrm{B}}$, $\mathrm{Co}_{9}$ 基态团簇的磁性大幅度的降低, 这是继 $\mathrm{Co}_{5}$ 之 后又出现的一个磁性局域最小点. $\mathrm{Co}_{10}$ 基态团簇的 磁性与 $\mathrm{CO}_{8}$ 完全相同, 这主要是由于在 $\mathrm{Co}_{10}$ 团簇中 有一个 $\mathrm{Co}_{6}$ 的八面体母体, 在八面体母体中有两个 Co-Co 键长达到 $0.3231 \mathrm{~nm}(\mathrm{GGA}), 0.3199 \mathrm{~nm}$ (LSDA), 如图 2 所示, 其值超出 Co-Co 间的成键范 围, 原子间的波函数交叠较小, 磁性有增大的趋势, 而配位数增大又使磁性减小, 两种因素相互作用最 终导致其磁性在 $\mathrm{Co}_{8}$ 基态的基础上没有发生变化.

在同一团簇中, 原子的配位数越小, 局域磁矩就 越大, 如表 2 中 $\mathrm{Co}_{5}$ 团簇三角双雉两个顶端原子的 磁矩就大于其它三个原子的磁矩; 局域配位数越大, 局域磁矩就应该越小, 如 $\mathrm{Co}_{10}$ 团簇八面体的两个顶 端原子配位数为 7 , 大于其它原子, 它的局域磁矩均 小于其它 8 个原子. 但也有例外, 如在 $\mathrm{Co}_{9}$ 基态团 簇中, 柱面上的三个帽原子尽管配位数 4 小于棱柱 原子的配位数 5 , 其帽原子磁矩 $1.41 \mu_{\mathrm{B}}$ (GGA) 和 1. $41 \mu_{\mathrm{B}}$ (LSDA) 小于棱柱原子的磁矩 $1.80 \mu_{\mathrm{B}}$

表 $4 \operatorname{Co}_{n}(n=2 \sim 10)$ 团簇的平均配位数 $(\mathrm{NC})$ 和平均键长 $(\bar{l})$

Table 4 Average coordination numbers $(\mathrm{NC})$ and bond lengths $(\bar{l})$ the ground state structures of of $\operatorname{Co}_{n}(n=2 \sim 10)$ clusters

\begin{tabular}{|c|c|c|c|c|c|c|c|c|c|c|}
\hline & & $\mathrm{Co}_{2}$ & $\mathrm{Co}_{3}$ & $\mathrm{Co}_{4}$ & $\mathrm{Co}_{5}$ & $\mathrm{Co}_{6}$ & $\mathrm{Co}_{7}$ & $\mathrm{Co}_{8}$ & $\mathrm{Co}_{9}$ & $\mathrm{Co}_{10}$ \\
\hline \multicolumn{2}{|c|}{ Average $\mathrm{NC}(\mathrm{GGA})$} & 1.0 & 2.0 & 3.0 & 3.6 & 4.0 & 4.3 & 4.5 & 4.7 & 5.2 \\
\hline & (LSDA) & 1.0 & 2.0 & 3.0 & 3.6 & 4.0 & 4.3 & 4.5 & 4. 7 & 5.2 \\
\hline \multirow[t]{2}{*}{$\bar{l} / \mathrm{nm}$} & (GGA) & 0.2125 & 0. 2386 & 0. 2295 & 0. 2336 & 0.2303 & 0.2340 & 0.2356 & 0.2347 & 0. 2433 \\
\hline & (LSDA) & 0.1949 & 0.2172 & 0.2234 & 0.2250 & 0.2247 & 0.2277 & 0.2274 & 0.2274 & 0.2361 \\
\hline
\end{tabular}


表 $5 \operatorname{Co}_{n}(n=2 \sim 10)$ 团簇的 $\boldsymbol{E}_{n} 、 \boldsymbol{E}_{\mathrm{b}} 、 \Delta^{2} \boldsymbol{E}_{n}($ a. u. $)$

Table 5 Total energy, binding energies and $\Delta^{2} E_{n}$ of the ground state structures of $\operatorname{Co}_{n}(n=2 \sim 10)$ clusters

\begin{tabular}{|c|c|c|c|c|c|c|c|}
\hline \multicolumn{4}{|c|}{ GGA } & \multicolumn{4}{|c|}{ LSDA } \\
\hline Cluster & $E_{n}$ & $E_{\mathrm{b}}$ & $\Delta^{2} E_{n}$ & Cluster & $E_{n}$ & $E_{\mathrm{b}}$ & $\Delta^{2} E_{n}$ \\
\hline $\mathrm{Co}_{2}$ & -2765.8677 & 0.1019 & & $\mathrm{Co}_{2}$ & -2760.5528 & 0.1093 & \\
\hline $\mathrm{Co}_{3}$ & -4148.8858 & 0. 1299 & 0. 0499 & $\mathrm{Co}_{3}$ & -4140.9436 & 0.1474 & 0.0788 \\
\hline $\mathrm{Co}_{4}$ & -5531.8539 & 0. 1315 & -0.0245 & $\mathrm{Co}_{4}$ & -5521.2556 & 0. 1468 & -0.0383 \\
\hline $\mathrm{Co}_{5}$ & -6914.8464 & 0. 1373 & -0.0374 & $\mathrm{Co}_{5}$ & -6901.6060 & 0. 1540 & -0.0724 \\
\hline $\mathrm{Co}_{6}$ & -8287.8764 & 0.1474 & 0.0298 & $\mathrm{Co}_{6}$ & -8282.0287 & 0. 1709 & 0.0639 \\
\hline $\mathrm{Co}_{7}$ & -9680.8766 & 0. 1504 & -0.0279 & $\mathrm{Co}_{7}$ & -9662.3875 & 0. 1739 & -0.0285 \\
\hline $\mathrm{Co}_{8}$ & -11063.9047 & 0.1561 & 0.0163 & $\mathrm{Co}_{8}$ & -11042.7748 & 0. 1796 & -0.0079 \\
\hline $\mathrm{Co}_{9}$ & -12446.9165 & 0. 1587 & 0.2265 & $\mathrm{Co}_{9}$ & -12423.1700 & 0.1851 & 0.0439 \\
\hline $\mathrm{Co}_{10}$ & -13829.9056 & 0. 1586 & & $\mathrm{Co}_{10}$ & -13803.5214 & 0. 1850 & \\
\hline
\end{tabular}

(GGA) 和 $1.80 \mu_{\mathrm{B}}(\mathrm{LSDA})$, 这主要是由于与帽原子 相互成键的键比较集中, 致使帽原子的 $d$ 电子较多 地参与了成键的电子, 磁矩变小; 反之, 如果原子相 互成键比较分散, 尽管配位数大, 仍能获得较大的 磁矩. 如 $\mathrm{CO}_{7}$ 团簇五角双雉的两个顶端原子, 其原 子配位数为 5 , 双雉其它五个原子的配位数是 4 , 但 其磁矩为 $2.27 \mu_{\mathrm{B}}$ (GGA) 和 $2.22 \mu_{\mathrm{B}}$ (LSDA) 却大 于其它五个原子的磁矩 $2.09 \mu_{\mathrm{B}}$ (GGA) 和 $2.11 \mu_{\mathrm{B}}$ (LSDA).

\section{4 团簇的相对稳定性}

表 5 列出了由 GGA 和 LSDA 两种方法计算得 到的总能量. 为了进一步了解 $\mathrm{Co}_{n}(n=2 \sim 10)$ 团 簇的最低能量的几何构型稳定性的变化规律, 我们 从如下的反应定义能量 $E_{\mathrm{b}}$, 以及总能量的二次差分 值 $\Delta^{2} E_{n}$ :

$$
\begin{aligned}
& \mathrm{Co}_{n} \rightarrow n \mathrm{Co} \\
& E_{\mathrm{b}}=E-1 / n E_{n} \\
& \Delta^{2} E_{n}=E_{n-1}+E_{n+1}-2 E_{n}
\end{aligned}
$$

式 (2) 中 $E_{\mathrm{b}}$ 为 $\mathrm{Co}_{n}$ 团簇分解为 $\mathrm{Co}$ 原子时的平均结 合能, $E$ 为一个 $\mathrm{Co}$ 原子的能量, $E_{n}$ 为 $\mathrm{Co}_{n}$ 团簇的 总能量. $E_{\mathrm{b}}$ 与 $n$ 的关系曲线如图 3 所示. 随着 $n$

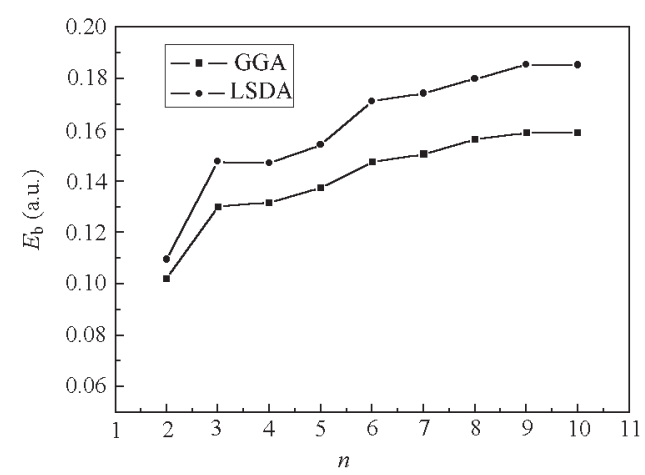

图 $3 \operatorname{Co}_{n}(n=2 \sim 10)$ 团簇 $\boldsymbol{E}_{\mathrm{b}}$ 与 $\boldsymbol{n}$ 的关系

Fig. $3 \boldsymbol{E}_{\mathrm{b}}$ of $\operatorname{Co}_{n}(n=2 \sim 10)$ clusters versus $n$

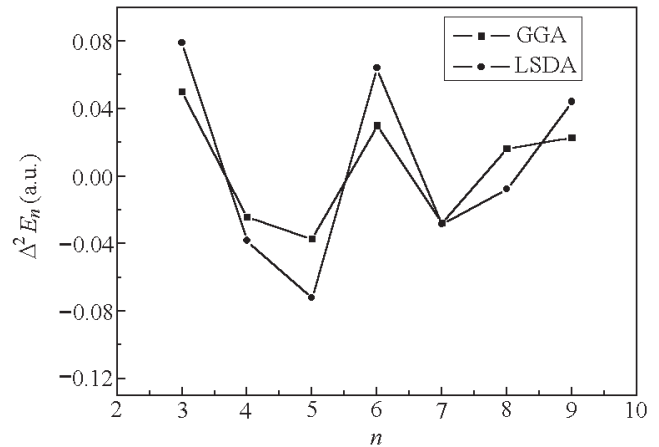

图 $4 \operatorname{Co}_{n}(n=2 \sim 10)$ 团簇 $\Delta^{2} \boldsymbol{E}_{n}$ 与 $\boldsymbol{n}$ 的关系

Fig. $4 \Delta^{2} E_{n}$ of $\operatorname{Co}_{n}(n=2 \sim 10)$ clusters versus $n$

的增大, $E_{\mathrm{b}}$ 在逐渐地增大, 而且有收玫于一点的趋 势, 在 $n=9 、 10$ 时, 两种方法给出的平均结合能几 乎完全相同. 式 (3) 中 $E_{n} 、 E_{n-1}$ 和 $E_{n+1}$ 分别为 $\mathrm{Co}_{n} 、 \mathrm{Co}_{n-1}$ 和 $\mathrm{Co}_{n+1}$ 基态团簇的总能量. $\Delta^{2} E_{n}$ 与 $n$ 的关系曲线如图 4 所示. 随着 $n$ 值的增大, 曲线没 有奇偶交替出现, 在 $n=3$ 出现了峰值, 以后每隔两 个点出现一个峰值, 即 $n=6 、 9$ 相继出现了峰值, 存 在峰谷交替, 而且两种方法的平行性相当好。在图 4 中, $n=3 、 6$ 和 9 这些 3 的整数倍时, $\Delta^{2} E_{n}$ 处于峰 值, 说明在 $\mathrm{Co}_{n}(n=2 \sim 10)$ 团簇中, $\mathrm{Co}_{3} 、 \mathrm{Co}_{6} 、 \mathrm{Co}_{9}$ 的 相对稳定性要大于其它团簇. 最稳定的是 $\mathrm{Co}_{3}$ 团 簇, $\mathrm{Co}_{6}$ 次之, $\mathrm{Co}_{6}$ 团簇的稳定性与 Mlynarski ${ }^{[10]}$ 和 Fan ${ }^{[11]}$ 所得结论相吻合. 最不稳定的团簇是 $\mathrm{Co}_{5}$ 团 簇, $\mathrm{Co}_{7}$ 次之. 这些结果为下一步在实验上合成和 理论上研究更大的团簇提供了一些理论依据.

\section{3 结 论}

1) 在 GGA 和 LSDA 两种方法下, 我们所确定 的基态构型完全一致, $n=2 、 3$ 为线性和平面结构, $n \geqslant 4$ 为紧密堆积的三维结构.

2 ) $\mathrm{Co}_{n}(n=2 \sim 10)$ 基态团簇的磁性在 $n=5$ 和 
9 出现了磁性局域最小点, 且基态团族的磁性在 $n=2 \sim 4$ 主要受平均键长的影响, 在 $n=5 \sim 9$ 主要 受平均配位数的影响, 在 $n=10$ 受原子间距和平均 配位数的相互影响, 最终导致与 $\mathrm{Co}_{8}$ 基态团簇具有 相同的磁性.

3 ) 在 $\mathrm{Co}_{n}(n=2 \sim 10)$ 基态团簇中, $\mathrm{Co}_{3} 、 \mathrm{Co}_{6}$ 和 $\mathrm{Co}_{9}$ 的相对稳定性要大于其它团簇. 最稳定的是 $\mathrm{Co}_{3}$ 团簇, $\mathrm{Co}_{6}$ 次之, 最不稳定的是 $\mathrm{Co}_{5}$ 团簇, $\mathrm{Co}_{7}$ 次之.

\section{References}

1 Salahub, D. R. ; Messmer, R. P. Surf. Sci., 1981, 106: 415

2 Lee, K. ; Callaway, J. ; Dhar, S. Phys. Rev. B, 1985, 30: 1724

3 Rosen, A. ; Rantala, T. T. Z. Phys. D, 1986, 3: 205

4 Pastor, G. M. ; Dorantes-Davila, J. ; Benneman, K. H. Phys. Rev. B, 1989, 40: 7642

5 Fujima, N. ; Yamaguchi. T. J. Phys. Soc. Jpn., 1989, 58: 3290

6 Dunlap, B. I. Phys. Rev. A, 1990, 41: 5691

7 Deheer, W. A. ; Milani, P. ; Chatelain, A. Phys. Rev. Lett., 1990, 65: 488
8 Bucher, J. P. ; Douglass, D. C. ; Bloomfield, L. A. Phys. Rev. Lett., 1991, 66: 3052

9 Louderback, J. G. ; Cox, A. J. ; listing, L. J. ; Douglass, D . C. ; Bloomfield, L. A. Z. Phys. D, 1993, 26: 301

10 Mlynarski, P. ; Iglesias, M. ; Pereiro, M. ; Baldomir, D. ; Wojtczak, L. Vacuum, 1999, 54: 143

11 Fan, H. J. ; Liu, C. W. ; Liao, M. S. Chem. Phys. Lett., 1997, 273: 353

12 Li, Z, Q. ; Gu, B. L. Phys. Rev., 1993, 47: 13611

13 Duan, H. M. ; Zhang, Q. O. Phys. Lett. A, 2001, 280: 333

14 Webber, S. E. ; Rao, B. K. ; Jena, P. ; Stepanyuk, V. S. ; Hergert, W. ; Wildberger, K. ; Zeller, R. ; Dederichs, P. H. J. Phys. : Codens. Matter, 1997, 9: 10739

15 Vosko, S. H. ; Wilk, L. ; Nusair, M. Can. J. Phys., 1980, 58: 1200

16 Becke, A. D. J. Chem. Phys., 1998, 88: 1053

17 Perdew, J. P. ; Wang, Y. Phys. Rev. B, 1992, 54: 13244

18 Bouarab, S. ; Vega, A. ; López, M. J. ; Iňiguez, M. P. ; Alonso, J. A. Phys. Rev. B, 1997, 55: 13279

19 Andriotis, A. N. ; Meonon, M. Phys. Rev. B, 1998, 57: 10069

\title{
Structure and Magnetism of $\mathrm{Co}_{n}(n=2 \sim 10)$ Clusters*
}

\author{
Lü Jin Xu Xiao-Hong Wu Hai-Shun \\ ( School of Chemistry and Material Science, Shanxi Normal University, Linfen 041004)
}

\begin{abstract}
All geometry structures of $\mathrm{Co}_{n}(n=2 \sim 10)$ clusters have been optimized, and the energy, frequence and magnetism of $\mathrm{Co}_{n}(n=2 \sim 10)$ clusters were calculated using the local spin density approximation and generalized gradient approximation of density functional theory. We found the same ground state structures in the two methods, and discussed theoretically the influence of the average coordination number, average bond length and symmetry on clusters magnetism. For $\mathrm{Co}_{n}(n=2 \sim 4)$ clusters, the magnetism of ground states strongly depends on average bond length, for $\mathrm{Co}_{n}(n=5 \sim 9)$, it strongly depend on average coordination number, for $\mathrm{Co}_{10}$, the factors of interatomic distance and average coordination number together lead to the same magnetism as Co ${ }_{8}$ cluster. Average moments of $\mathrm{Co}_{5}$ and $\mathrm{Co}_{9}$ present magnetic magic number.
\end{abstract}

Keywords: Clusters, Structure of ground state, Magnetic moment, Average bond length, Average coordination number, Symmetry 